
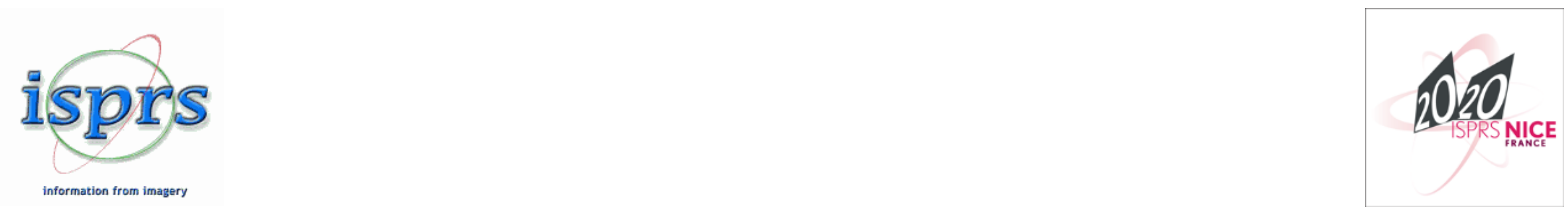

\title{
Preface
}

\section{Technical Commission II}

ISPRS Technical Commission II focuses, at various scales, on geometric, radiometric and multi-temporal aspects of image- and range-based 3D surveying and modeling. Specifically, Commission II deals with image orientation, point cloud generation and processing, 3D feature extraction, scene understanding, sensor and data fusion, sensor characterization, machine learning for geospatial data analysis and big data techniques for massive data processing. Applications in the fields of mapping, industry, heritage, space, underwater and environment are considered.

The Volume related to Commission II contains 182 papers published in the ISPRS Archives and 118 published in the ISPRS Annals. The Archives were accepted on the base of an abstract review from originally submitted 262 abstracts. The Annals comprise reviewed articles accepted from the originally submitted 191 full papers.

Considering the aforementioned research issues, challenges and needs, the papers published depict the latest developments in methodological aspects and the most interesting applications in photogrammetry.

Great interest was dedicated to machine/deep learning methods used to solve various steps of the photogrammetric pipeline and in particular for the semantic enrichment of images and 3D data. Many papers reported new models and methods to extract features, geometrical primitives and objects from data acquired by airborne and/or terrestrial sensors, including object recognition and 3D object/scene reconstruction.

Various contributions referred to the utilization, integration, modeling and performance analyses of imaging and ranging sensors in the industry sector, environment and heritage fields. Cultural Heritage (including Underwater) attracted many submissions with various worldwide applications which depicted how 3D documentation is increasingly used and crucial for conservation, preservation and valorisation needs.

We believe these volumes nicely recap the state of the art, current trends and possible applications in photogrammetry, with a nice overlook at the forthcoming years of investigations.

On behalf of Technical Commission II, we would like to thank the local organisers of the 2020 ISPRS Congress, the members of the international program committee, all Working Group officers and all reviewers for their hard organizational work and efforts in the paper reviewing process. 